\title{
El consentimiento informado en la Seguridad Social en Costa Rica. Impacto de las sentencias judiciales
}

Informed consent in Social Security in Costa Rica. Impact of court rulings.

\section{Alejandro A. Marín Mora}

Médico, MSc. Centro de Desarrollo Estratégico e Información en Salud y Seguridad Social de la Caja Costarricense de Seguro Social. San José, Costa Rica.

Palabras clave: Consentimiento informado; Reglamento de Consentimiento Informado en la Práctica Asistencial de la Caja Costarricense de Seguro Social; Ética.

Key words: Informed consent; regulation (Costa Rica); ethics.

La doctrina del consentimiento informado se ha convertido en un punto focal en las discusiones sobre la información terapéutica y la controversia que genera la discrecionalidad del médico en cuanto a la profundidad y claridad de la información, sobre todo de los riesgos de las intervenciones que pretende realizar sobre el paciente. Asimismo los sistemas sanitarios valoran cada vez más el impacto de las sentencias judiciales sobre sus procesos y el costo económico que dichas sentencias exigen para su cumplimiento.

En Costa Rica dos sentencias ponen en la palestra la unión de ambos temas.

El tema de consentimiento informado no es extraño para nuestro país, tanto para los médicos como para los abogados. En la Constitución Política (Costa Rica, 1949), en sus numerales 24.- y 27.-, se acopian las premisas para fundamentar el consentimiento informado, el cual queda plasmado, en el año 1973, con la Ley General de Salud (Ley $N^{\circ}$ 5395) específicamente en sus a rtículos 10.- y 22.- (Costa Rica, 1973), y vuelve a reiterarse en el año 2002 en la Ley Derechos y Deberes de las Personas Usuarias de los Servicios de Salud Públicos y Privados de Costa Rica (Costa Rica, 2002).

Además, puede encontrarse la obligatoriedad legal de aplicar el consentimiento informado en otros distintos cuerpos normativos (algunos de mayor data que los citados anteriormente) entre los que destacan, a nivel de ley, el Código Civil, el Código Penal, el Código de Familia y el Código de la Niñez y la Adolescencia, y, a 
nivel reglamentario, el recién aprobado Reglamento de Consentimiento Informado en la Práctica Asistencial de la Caja Costarricense de Seguro Social (CCSS, 2012).

Por último, el término puede encontrarse en distintos códigos de ética de colegios profesionales en ciencias de la salud, entre los que destaca el del Colegio de Médicos y Cirujanos de Costa Rica (Costa Rica, 2009).

Es por ello que, existiendo todo un cuerpo normativo que obligue al cumplimiento de tan importante derecho - entendiendo el consentimiento informado como un derecho - despierta la interrogante sobre la Sentencia de la Sala Primera de la Corte Suprema de Justicia no. 1102-F-S1-2010, de las 14:50 horas del 14 de septiembre de 2010 (Costa Rica, 2010), en donde se demostró el no cumplimiento íntegro de este derecho por quienes estén llamados a otorgarlo (los profesionales en salud), convirtiéndose en la más clara sentencia condenatoria para la Caja Costarricense de Seguro Social en este tema.

Resulta cierto e indiscutible que el consentimiento informado es, por tanto, la justificación misma de la legitimidad del acto médico o clínico, basado en el derecho de la persona a su autonomía o autodeterminación, cuando cuente con capacidad y competencia para ejercerla.

Para la jurisprudencia, el consentimiento informado trae consigo la asunción por el paciente de los riesgos y secuelas inherentes o asociados al procedimiento clínico autorizado; pero no excluye la responsabilidad médica por daños que son imputables a una actuación negligente del médico o al mal funcionamiento del centro asistencial (Gargallo, 2004).

Aparte de legitimar el acto médico, el consentimiento informado trae consigo una serie de beneficios que deben ser conocidos por los médicos, con el fin de garantizar su correcta aplicación: garantiza al paciente su derecho a ejercer su autonomía y su voluntad; permite tomar decisiones sobre las acciones de salud en un marco de confianza y apoyo, mejorando así la adherencia a los tratamientos y fomentando el autocuidado de la salud, y, por último, brinda mayor seguridad jurídica al proceso de atención en salud.

\section{El consentimiento informado en la Seguridad Social en Costa Rica}

Entre los antecedentes de mayor importancia se encuentra el Informe Extraordinario de Labores, presentado en marzo del 2001, en el que la Defensoría de los Habitantes de Costa Rica se pronunció sobre el escenario de desprotección que 
afrontan las personas con discapacidad mental para comprender las intervenciones clínicas a los que podían ser sometidos, como es el caso de las esterilizaciones. Según informe final Oficio № 03511 (2001), se estipulo una recomendación para la Caja Costarricense de Seguro Social (CCSS):

[...] girar instrucciones a los centros de salud, a efecto de contemplar las necesidades particulares de las personas con discapacidad, particularmente en cuanto a información sobre los derechos reproductivos y sexuales, en el caso de que las personas con discapacidad no puedan comprender la trascendencia de la esterilización [...] (Costa Rica, 2001).

La Defensoría encomendó además al Hospital Nacional Psiquiátrico (CCSS) lo siguiente: "[...] Informar al paciente menor de edad sobre los tratamientos que eventualmente se le podría suministrar para oír su criterio, el cual debe ser valorado de conformidad con su edad y su madurez", solicitud que se fundamentó en el derecho de los niños, niñas y adolescentes a "[...] Expresar su opinión en los ámbitos de su vida cotidiana, especialmente en la familia, la comunidad y la escuela; como usuario de todos los servicios de salud y, con las limitaciones de la ley, en todos los procesos judiciales y administrativos que puedan afectar sus derechos" (Costa Rica, 2001).

Para este entonces la CCSS contaba con un formulario denominado Autorización y Exoneración de Responsabilidad para Tratamientos y Procedimientos Médicos y Quirúrgicos. La Defensoría consideró que dicho documento no contenía ninguna indicación acerca de las circunstancias del acto como la fecha, hora, los nombres de los testigos y, los más importante, no se indicaba qué información que se le ofrecía a la persona. La Defensoría concluyo que, independientemente de que dicho formulario se encontrara lleno, no contenía las exigencias del derecho a la información y no desarrolla, por lo tanto, las exigencias del consentimiento informado. Por lo tanto, no constituía prueba fehaciente de que a las personas usuarias se les brindara información y que se recogiera en éste el consentimiento requerido para los distintos tipos de intervención médica que se pueden producir (Costa Rica, 2001).

Finalmente, como se sabe, los profesionales en salud no pueden, mediante este tipo de "formularios de exoneración", eximirse de responsabilidad civil o penal por una actuación imprudente, negligente o por no contar con la pericia respectiva. Es así que el consentimiento informado no es un elemento que libera a los profesionales de la salud de responder por los casos de negligencia, imprudencia o impericia, dado 
que el derecho a la información a la persona no guarda relación alguna con la responsabilidad penal del ejercicio legal de su profesión.

Posteriormente a estas recomendaciones, la CCSS oficializo un formulario (CCSS, s/d), el cual se utiliza hoy en día a nivel en dicha institución. Este formulario, según demanda el recién aprobado Reglamento de Consentimiento Informado en la Práctica Asistencial de la Caja Costarricense de Seguro Social, será reemplazado para dar lugar a un Catálogo de Formularios de Consentimiento Informado, siguiendo el modelo de la Junta de Andalucía (España).

\section{A razón de las sentencias judiciales}

La primera de las dos sentencias que analizaremos es la Sentencia de la Sala Primera de la Corte Suprema de Justicia no. 1102-F-S1-2010, que condenó a la Caja Costarricense de Seguro Social a la indemnización al señor "LIVC", por un monto de 40.000 dólares americanos.

Dicho paciente quien fuere internado en el Servicio de Neurología del Hospital Dr. Rafael A. Calderón Guardia y al cual, el 26 de diciembre de 2000, se le intervino quirúrgicamente por una compresión medular a nivel de la vértebra torácica T7, resultando de una complicación post operatoria, denominada paraplejia.

La sentencia se fundamentó no en la paraplejia sufrida sino en la falta de información al demandante sobre los riesgos de la cirugía, lo que, en su criterio, le limitó su derecho a decidir si se sometía o no a la operación. Todo ello basado en los ordinales 22 de la Ley General de Salud que estipula: "Ninguna persona podrá ser sometida a tratamiento médico o quirúrgico que implique grave riesgo para su integridad física, su salud o su vida, sin su consentimiento previo o el de la persona llamada a darlo legalmente si estuviere impedido para hacerlo. Se exceptúa de este requisito las intervenciones de urgencia" (Costa Rica, 1973). También, la Ley No. 8239 (Ley de Derechos y Deberes de las Personas Usuarias de los Servicios de Salud Públicos y Privados de Costa Rica), de 19 de abril de 2002, en el precepto $2^{\circ}$, de manera taxativa entre los derechos de los pacientes incluye: “...b) recibir la información necesaria y, con base en ella, brindar o no su autorización para que les administren un determinado procedimiento o tratamiento médico" (Costa Rica, 2002).

De ahí que toda persona usuaria de los servicios de salud públicos o privados tiene derecho a que se le informe en detalle de su padecimiento, posibles tratamientos o intervenciones quirúrgicas a aplicar y sus riesgos, señala la sentencia: 
[...] la obligación de informar es un elemento esencial de la "lex artis ad hoc" [...] En lo referente a la carga de la prueba del cumplimiento del deber de información, es claro que le concierne al profesional de la medicina, ya que se encuentra en una situación más favorable para acreditarlo [...] En estas circunstancias, no basta con que al paciente se le haga firmar un documento, sin importar el nombre que se le otorgue, lo primordial es demostrar que se le informó de manera clara, precisa y en términos comprensibles los peligros que entrañaba la cirugía. (Costa Rica, 2010)

De la sentencia se rescatan cuatro elementos vitales: primero, la carga de la prueba corresponde al médico; segundo, que resulta imperativo informar sobre las consecuencias que conllevan las intervenciones clínicas; tercero, demostrar que se informó al paciente de manera comprensible y adecuada a sus capacidades, y, último, que la sola firma de un documento no basta para los propósitos del consentimiento informado.

Como puede observarse, pese al costo económico que representó la sentencia para la Caja Costarricense de Seguro Social, resultó evidente la violatoria al derecho a la información, el cual es una derivación del derecho humano a la autonomía de la voluntad o a la libre determinación de las personas como seres autónomos.

En cuanto a la segunda sentencia, esta de la Sala Constitucional de la Corte Suprema de Justicia (Costa Rica, 2013), ordena a la Caja Costarricense de Seguro Social a informar a los pacientes cuando se les pretenda cambiar de un medicamento de marca al mismo pero en su versión de medicamento genérico.

Esto según el análisis que hicieron los magistrados, tras el caso de un recurso de amparo presentado ante ellos por los pacientes a los que se les administraba un medicamento de marca cuando, en el servicio de nefrología pediátrica del Hospital Nacional de Niños de Costa Rica, se intentó realizar el cambio a un medicamento genérico.

Si bien los magistrados no encontraron que el cambio al medicamento genérico hubiera causado la afectación a la salud que los recurrentes argumentaban, ordenaron en la sentencia que, en el futuro, todo cambio de medicamento de marca a genérico debe llevar previo un consentimiento informado.

Esta sentencia, además de que se contrapone a las estrategias de medicamentos genéricos impulsada por la Organización Mundial de la Salud (OPS, 2011) como búsqueda para mejorar el acceso a los medicamentos, ocasionará en el mediano plazo, un coste económico inimaginable para la Seguridad Social del país, ya que obliga a la CCSS a mantener líneas de medicamentos de marca para aquellos 
pacientes que no consientan el cambio a un medicamento genérico, en detrimento del principio de justicia y de la sostenibilidad financiera del sistema de salud público del país.

De las interrogantes sobre esta sentencia, surgen las siguientes, ¿cómo proceder si el fabricante eleva desproporcionadamente los costos del medicamento de marca y el paciente no consiente el cambio a genérico?, o bien, ¿cómo actuar si el fabricante retira del mercado el medicamento de marca?, estos planteamientos llevan a preguntarse sobre el costo de dicha sentencia representa para la CCSS, el cual a la fecha es incalculable.

Debido a que la teoría sobre el consentimiento informado dictamina que este debe ser solicitado antes de la administración de un medicamento, o previo a la realización de un procedimiento quirúrgico, no ha sido posible encontrar evidencia teórica para justificar dicha sentencia y demostrar la necesidad de un consentimiento para este caso en particular (el cambio de medicamento de marca a genérico), ya que estamos frente a compuestos químicos idénticos y en donde no hay un cambio real de terapéutica, de dosificación, de vía de administración o de compuesto químico, en donde, si, entraría un nuevo consentimiento a ser protagonista, sino se está frente a un meramente cambio de nombre: de marca o comercial a genérico.

\section{Conclusión}

Resulta fundamental, como puede deducirse, una mayor capacitación y divulgación entre los médicos de temas sobre derechos y deberes de los pacientes, de manera que perciban el tema de consentimiento informado como un aliado más en la práctica sanitaria; que los pacientes exijan respetuosamente su derecho concebido en la ley; y que las autoridades y dirigentes hospitalarios realicen los cambios en los procesos y modelos asistenciales para dar mayor espacio al tema de consentimiento informado, ya que si esto último no se realiza, será concebido como un mero trámite administrativo.

Debido a que la medicina no es una ciencia exacta y, por tanto, los resultados no son exigibles, el profesional de la salud deberá poner al servicio de su paciente sus conocimientos científicos, recursos técnicos y tiempo necesario, así como brindar información completa, relevante, veraz, pertinente, clara y entregada de manera oportuna. 
Esto queda demostrado con la sentencia de la Sala Primera sobre el caso de omisión al derecho de información que impidió al paciente tomar una decisión informada previa a un procedimiento quirúrgico.

Pero queda demostrada también la necesidad imperiosa de formar en Derecho Sanitario a los jueces y magistrados en temas como información terapéutica, consentimiento informado, privilegio terapéutico, voluntad anticipada y otros, con el fin de promover sentencias con una mejor fundamentación teórica que impida, por errores conceptuales, imponer sentencias a las instituciones sanitarias que les impliquen un costo elevado en detrimento de la sostenibilidad de la atención y de la garantía al derecho a la salud, como queda evidenciado en la sentencia de la Sala Constitucional de la Corte Suprema de Justicia.

\section{Referencias}

CAJA COSTARRICENSE DE SEGURO SOCIAL (CCSS). Reglamento del Consentimiento Informado en la Práctica Asistencial en la Caja Costarricense de Seguro Social. 2012 [on line] Disponible en: http://www.cendeisss.sa.cr/etica/REGLAMENTO-Cl-2012.pdf

CAJA COSTARRICENSE DE SEGURO SOCIAL (CCSS). Consentimiento Informado [formulario]. s/d. [on line] Disponible en: http://portal.ccss.sa.cr/portal/ page/portal/Gerencia_Medica/Proyeccion_Servicios_Salud/Estadistica_en_Salud/Doc umentos/TabExpedienteSalud/TabAdolescente/4-70-03-

0560_Consentimiento_General_2007.pdf

COSTA RICA. Constitución Política de Costa Rica (1949). [on line] Disponible en: http://pdba.georgetown.edu/Parties/CostaRica/Leyes/constitucion.pdf

COSTA RICA. Corte Suprema de Justicia. Sentencia no. 001102-F-S1-2010, de 14 sep. 2010. [on line] Disponible en: http://www.poderjudicial.go.cr/salaprimera/index.php/lista-de-noticias/171-noticia-conocimiento-riesgo

COSTA RICA. Corte Suprema de Justicia. Resolución no. 2013-015485, de 2013. [on line] Disponible en: http://sitios.poder-judicial.go.cr/ salaconstitucional/Actas\%20de\%20Votacion/2013/v112613b.pdf

COSTA RICA. Decreto No. 35 332-S, del 15 de mayo de 2009 (Código de Moral Médica). [on line] Disponible en: http://www.cendeisss.sa.cr/etica/cod-moral-etica.pdf .

COSTA RICA. Defensoría de los Habitantes de Costa Rica. Informe Extraordinario de Labores (Oficio No. 03511, del 2 de mayo de 2001). [on line] Disponible en: http://dhr.go.cr/la_defensoria/informes/labores/documentos/if00_01adicional.pdf .

COSTA RICA. Ley No. 5395 (Ley General de la Salud). Gaceta No. 222, del 24 de noviembre de 1973. [on line] Disponible en: http://www.msp.go.cr/ministerio/gestion\%20ambiental/normativa\%20aplicable $\% 20 y \%$ 20vigente/leyes/LEY\%20GENERAL\%20DE\%20SALUD\%20Ley\%20No\%205395.pdf

COSTA RICA. Ley No. 8239, del 2 de abril de 2002 (Derechos y Deberes de las Personas Usuarias de los Servicios Públicos y Privados de Costa Rica). Gaceta No. 
75, del 19 de abril de 2002. [on line] [Disponible en: http://www.ministeriodesalud.go.cr/gestores_en_salud/derechos\%20humanos/leyes/leyusuari ossalud.pdf ]

GARGALLO, I. S. Tratamiento legal y jurisprudencial del consentimiento informado. Working Paper $\mathrm{n}^{\circ}$ 209. Barcelona, 2004. Disponible en: http://www.indret.com/pdf/209_es.pdf

ORGANIZACIÓN PANAMERICANA DE LA SALUD (OPS). Guía para la implementación de estrategias de medicamentos genéricos en los países de América Latina y el Caribe como mecanismo para mejorar el acceso a medicamentos. Washington: Organización Panamericana de la Salud, 2011. ISBN 978-92-75-33223-8

Recebido para publicação em 5 de maio de 2014. Admitido para publicação em 23 de junho de 2014. 